. Revista Arbitrada Multidisciplinaria de Ciencias de la Salud. SALUD Y VIDA

Volumen 2. Número 4. Año 2. Julio - Diciembre 2018. Santa Ana de Coro, Venezuela.

Hecho el depósito de Ley: FA2016000010

ISSN: $2610-8038$

FUNDACIÓN KOINONIA (F.K). Santa Ana de Coro, Venezuela.

Sandra Romero

\title{
EI Médico ante los desafíos de la Salud en el Siglo XXI
}

The Doctor faced with the challenges of Health in the 21st Century

\author{
Sandra Romero \\ Universidad Nacional Experimental "Francisco de Miranda" \\ sandrar.unefm@gmail.com \\ Venezuela
}

Recibido: 3 de abril de 2018

Aprobado: 20 de mayo de 2018

\section{RESUMEN}

A nivel mundial se plantean retos importantes para el mantenimiento de la salud por lo que se establecen acciones desde los ámbitos políticos, económicos, sociales y hasta culturales para lograr el equilibrio entre la salud y la enfermedad de la población. Estos retos tienen en cuenta las transformaciones que ha sufrido la sociedad, los cuales consideran los cambios experimentados en la unidad fundamental como lo es la familia, teniendo en cuenta los aspectos económicos, las enfermedades emergentes, reemergentes y hasta las enfermedades desatendidas u olvidadas. Es por ello, que se requiere replantear la formación del médico como profesional ante estos nuevos desafíos de manera que a partir de sus conocimientos, habilidades, destrezas, competencias, es decir, a través de la calidad educativa y la pertinencia social de conocimiento, y en acción conjunta con un equipo interdisciplinario se pueda asumir la responsabilidad de implementar estrategias en lo que a salud se refiere, y dar respuesta eficaz y eficiente a las problemáticas de salud en la sociedad de hoy.

Descriptores: Salud; médicos; formación en competencias; calidad educativa; pertinencia social.

\section{ABSTRACT}

At a global level, important challenges are posed for the maintenance of health, which is why actions are established from the political, economic, social and even cultural spheres to achieve a balance between health and disease of the population. These challenges take into account the transformations that society has undergone, which consider the changes experienced in the fundamental unit such as the family, taking into account the economic aspects, emerging diseases, reemerging and even neglected or forgotten diseases. That is why it is necessary to rethink the training of the doctor as a 
professional in the face of these new challenges so that based on their knowledge, abilities, skills, competences, that is, through the educational quality and the social pertinence of knowledge, and in joint action with an interdisciplinary team can take responsibility for implementing strategies in terms of health, and give an effective and efficient response to health problems in today's society.

Descriptors: health; doctors; competency training; educational quality.

\section{INTRODUCCIÓN}

La salud como derecho fundamental del individuo se constituye en el propósito esencial de las diferentes naciones y de las organizaciones a nivel mundial, de tal manera quelas políticas públicas en salud aplicadas en este sentido, deben tener en cuenta las problemáticas que enfrentan las distintas regiones y especialmente en América Latina. Cabe señalar, que la salud pública ha venido evolucionando desde la época de Hipócrates, cuando se comenzó a hablar de la influencia del ambiente físico y social en la salud de la población. (OPS, 1992). Es por ello, que para Sigerist la salud pública tiene sus raíces en la religión, pues la limpieza era concebida con sentido espiritual, tal como se expresa en el libro Levítico, 1500 a.c, pero aclaran que los primeros momentos de la salud pública fueron en Roma antigua, cuando se conocieron con la denominación de Higiene Pública, que se dedicaba a los aspectos preventivos relacionados con el ambiente físico, en especial agua y acueductos. (Ramos, 2000).

De este modo, se tiene que la importancia de la salud de la población y lo que sus implicaciones han sido prioridad de los Estados desde tiempos remotos. Conviene destacar, que en muchos países de la América latina, la salud como cuestión pública durante los dos primeros tercios del siglo XIX, estuvo dominada por los enfoques miasmáticos y medio-ambientalistas los cuales no produjeron cambios sanitarios infraestructurales de peso, limitando de ese modo sus efectos en la mortalidad general, pero hacia finales del siglo la bacteriología moderna tomará la iniciativa, marcando profundamente la dinámica de muchos de los emprendimientos en materia de salud pública. (Armus, 2000). 
Cabe señalar, que durante el siglo XIX uno de los principales exponentes de la medicina social fue Rudolf Virchow, patólogo alemán muy conocido por sus hallazgos científicos y que a través de sus publicaciones defendió que la salud humana es un asunto de interés social directo, y que las condiciones sociales y económicas deben ser analizadas científicamente como causas de enfermedad. Para Virchow, estaba muy claro que la medicina debía ser una de las bases científicas de la organización social, y también fue partidario de crear un ministerio de sanidad y llevar a cabo una profunda reforma de la enseñanza (Coronel ,2017).

Por otra parte se tiene, que las reformas en materia de salud impulsadas en el siglo XX y especialmente en las décadas de los ochenta y noventa en América Latina, se orientaban a reducir la responsabilidad del Estado en materia de políticas sociales, entre las que se encuentra especialmente la política sanitaria, trasladándola a las personas individualmente y al sector privado como administrador privilegiado (Borgia, 2006).

En este sentido es importante señalar que en el nuevo siglo XXI, se han trazado agendas desde la Organización Mundial de Salud (OMS, en lo sucesivo), donde se busca implementar políticas de salud que buscan superar la concepción de la medicina curativa y se orientan los esfuerzos hacia políticas públicas donde se promueve la salud y la prevención de las enfermedades, para tener como propósito fundamental el bienestar de la población y mejorar su calidad de vida. Pero, la pobreza y sus consecuencias negativas en el desarrollo humano se constituyen enla vía para el incremento tanto en la prevalencia como en la incidencia de muchas de las enfermedades transmisibles, las cuales se encuentran ampliamente distribuidas por todo el mundo y que las afecciones que ocasionan aparecen con mayor frecuencia en los países con menor desarrollo socio-económico.

Por otra parte, se tiene que la OMS (2005), reconoce que la salud es una meta social y una responsabilidad que concierne a toda la sociedad y que cada vez es mayor la evidencia de que mejorar la coordinación y el liderazgo, para hacer frente a las dimensiones sociales de la salud en todos los procesos de elaboración de políticas 
públicas es posible, y que ello redunda en una mejor salud y un mayor acceso a la atención sanitaria. Además afirma que actuando sobre las causas sociales de la mala salud, los gobiernos estarán en buena situación para alcanzar sus objetivos de desarrollo. De tal manera que, son las condiciones sociales, económicas y las ambientales que afectan la salud de la gente, relacionándolos con enfermedades físicas, enfermedades mentales y mortalidad.

Cabe señalar, que en la Novena Conferencia Mundial de Promoción de la Salud se celebrada en Shanghai, China, en noviembre del 2016, se planteó como tema central; "La promoción de la salud en los objetivos de desarrollo sostenibles", lo que constituye la proyección del trabajo en los próximos años,y es por ello que durante esta conferencia fue emitida la Declaración de Shanghai sobre la promoción de la salud, en la Agenda 2030 para el desarrollo sostenible (OMS, 2016).

De tal manera que, la promoción de la salud ha sido una preocupación de instituciones políticas y sociales, y que ha existido conciencia de que es un factor esencial para el mejoramiento de la salud en todo el mundo, y de forma particular en América Latina, donde gran parte de los países que la integran enfrenta problemáticas de desarrollo y salud comunes. Por lo que la equidad y la superación de la pobreza son cruciales en la búsqueda de soluciones integrales y de alto impacto.

Por otra parte, es necesario tener en cuenta elementos importantes para el logro de la salud con equidad en la sociedad, por lo que la epidemiologia social investiga de manera explícita los determinantes sociales en la distribución de la salud, la enfermedad y el bienestar en las poblaciones, en vez de tratar dichos determinantes como un simple trasfondo de los fenómenos biomédicos (Kriege,2002). Por lo tanto, la epidemiologia social pretende conocer como los factores sociales afectan a la salud de la población (Benach, 2005).

Es por ello,que dentro la epidemiologia social se resalta la importancia de la familia, lo que ella representa y su influencia con respecto a los índices de morbilidad -mortalidad de una población determinada. Es importante subrayar, que la familia se constituye en 
una institución esencial para el bien común y las constituciones políticas de muchos Estados reconocen explícitamente su valor e importancia (Castro, 2015).

Por consiguiente, se tiene que la familia sigue siendo una institución importante para la persona y para el desarrollo de la sociedad, su valor no recae por las funciones que de ella se desprenden o por los servicios que preste o por la utilidad que puede generar, sino por su relevancia como institución, espacio donde la persona accede más al ser y escuela del más rico humanismo (Morandé, 2006).

Es importante señalar, que la familia ha sufrido numerosos cambios en cuanto a su estructuración y a su desenvolvimiento en los últimos años. Es por ello, que numerosas investigaciones exponen las enormes consecuencias, a distintos niveles, de la desestructuración familiar (Pliego, 2012). Cabe considerar, que desde una perspectiva económica es importante valorar el aporte fundamental de la familia y que los efectos de esta realidad pueden analizarse a partir de tres grandes tendencias sociodemográficas, estrechamente relacionadas entre sí: los efectos del divorcio para la economía, el descenso en la tasa de natalidad y el actual proceso de cambio en la estructura familiar. Lo cual repercute seriamente en la economía de las naciones y genera un impacto importante en el desarrollo sostenible (Dumont, 2004).

Por otra parte, el rol que desempeña la familia en la determinación de la salud constituye un tema que amerita mayor atención en el marco de un sistema de salud sustentado en la práctica de la medicina familiar. Por lo que la atención primaria contemplan objetivos y acciones relacionadas con el diagnóstico y la intervención familiar, así como el análisis de la situación de salud de la familia, aspecto que implica la consideración de la familia como unidad básica para la atención de salud individual y comunitaria (Litman, 1994).

En este sentido, aún son insuficientes los fundamentos teóricos que contribuyan a la consideración de la familia como elemento que participa en la determinación de la salud, a fin de concebirla como grupo de atención y análisis, y no como la vía o el lugar para la organización de las acciones. Conviene destacar, que existen factores que influyen en la salud de la familia, como por ejemplo, los recursos materiales, como son 
las condiciones de empleo, es decir, la situación laboral, los riesgos en el trabajo, tanto físicos como ergonómicos además del entorno psicosocial. También entre los factores que afectan la salud de la familia están la carga de trabajos no remunerados del hogar y del cuidado de las personas, el nivel de ingresos, la situación económica y patrimonial, la calidad de la vivienda, sus equipamientos, y el barrio o área de residencia y sus características (Benach, 2005).

De tal manera, que estos factores tienen un impacto en la salud de la familia e influyen en procesos psicosociales como son la falta de control, la autorrealización o las situaciones de estrés, así como en las conductas con influencia en la salud. Por otra parte, se tiene que numerosas investigaciones muestran las enormes consecuencias, a distintos niveles, de la desestructuración familiar, y que desde una perspectiva económica, es importante valorar el aporte fundamental de la familia como dimensión clave en la generación de capital humano y desarrollo integral.

Por consiguiente, una economía que funciona bien considera y responde a las demandas primarias de la familia y posibilita la justa distribución de los factores de producción, facilitando de ese modo que sus miembros logren alcanzar una calidad de vida que les permita cubrir sus necesidades fundamentales (Longman, 2011). De este modo, de tiene que aunque los servicios sanitarios, en sí mismos, no son el factor principal de generación de las desigualdades en salud, pueden tener un efecto en estas, sobre todo si el acceso y la calidad a ellos no es equitativo para toda la población.

Conviene destacar, que las desigualdades a nivel de la salud son debidas a las diferencias que existen entre los grupos socioeconómicos, las cuales son sistemáticas, producidas socialmente además de injustas (Whitehead, 2006).Se debe señalar, que las diferencias son sistemáticas porque no se producen de forma aleatoria, sino que tienen un patrón persistente en la población, y afectan a los grupos más vulnerables. Se plantea que son consecuencia de procesos sociales y no de procesos biológicos, porque, las desigualdades en salud no están relacionadas, con factores hereditarios, sino que lo están con el funcionamiento de la sociedad. 
En este sentido, pueden presentarse como injustas para algunos grupos sociales porque vulneran derechos fundamentales de las personas. Es por ello, que la Comisión sobre Determinantes Sociales de la Salud de la Organización Mundial de la Salud, sostiene que la injusticia social está acabando con la vida de muchísimas personas y también afirma que las desigualdades en salud son el resultado de la situación en que la población crece, vive, trabaja y envejece, y del tipo de sistemas que se utilizan para combatir la enfermedad. A su vez, las condiciones en quela gente vive y muere están determinadas por fuerzas políticas, sociales y económicas (Borrell, 2012).

En cambio, conviene poner de relieve que ante esta problemática y los aciertos y desaciertos de las políticas públicas, y atendiendo a las nuevas exigencias que demanda la sociedad de hoy en atención de salud, especialmente la relacionada con la salud de la familia y lo que la afecta, se debe tener en cuenta la formación del talento humano para dar respuesta a los retos que se plantean desde el orden social para lograr el bienestar de la población y mejorar su calidad de vida. En este sentido, se tiene que el médico del siglo XXI debe poseer no solo conocimientos sino que debe tener una formación integral que le permita tener habilidades, destrezas y competencias para que actuando desde un ámbito interdisciplinario y transdiciplinario pueda dar solución a las problemáticas de salud que enfrenta la sociedad de hoy.

En esta perspectiva, la Conferencia Regional de América Latina y El Caribe (2008), declara que:

La educación general, y la superior en particular, son instrumentos esenciales para enfrentar exitosamente los desafíos del mundo moderno y para formar ciudadanos capaces de construir una sociedad más justa y abierta, basada en la solidaridad, el respeto por los derechos humanos y el uso compartido del conocimiento y la información. La educación superior constituye un elemento insustituible tanto para el desarrollo social, la producción, el crecimiento económico y el fortalecimiento de la identidad cultural, como para el mantenimiento de la cohesión social, la lucha contra la pobreza y la promoción de la cultura de la paz.

Cabe señalar, que se requiere en este momento histórico, la formación de profesionales con pertinencia social y calidad académica para poder lograr el desarrollo sostenible y 
sustentable del mundo de hoy. Por lo tanto, la educación superior tiene que asumir un papel importante porque debe ser el promotor del cambio social que conlleve a la generación de conocimientos que permitan el abordaje de las problemáticas a nivel mundial, específicamente en aspectos como la seguridad alimentaria, el cambio climático, la gestión del agua, el diálogo intercultural, las energías renovables y la salud pública (UNESCO, 1998).

En consecuencia, se ratifica la necesidad que se tiene de que las universidades e institutos y colegios universitarios trabajen de cara al contexto y al momento histórico con sentido de pertinencia, de apertura a nuevas formas de aprendizaje que se correspondan con: las características de vida del trabajador; las modalidades curriculares concretamente flexibles, que se ofrezcan de manera continua y permanente para mejorar y actualizar la formación profesional; la superación de la condición de estancos en la organización curricular; la aceptación de que lo inter y lo transdisciplinario es esencial para comprender la compleja realidad que se vive; la idea de que el conocimiento disciplinario es importante(UNESCO, 1998).

Igualmente, Morín (2007), plantea que la realidad cualquiera que sea física, biológica, psicológica, sociales complicada y como tal requiere un pensamiento complejo para ser comprendida y explicada, teniendo en cuenta sus procesos de orden, desorden y reorganización constante. Lo cual implica el reto de cambiar las formas y procesos de enseñanza-aprendizaje de los fenómenos laborales, sociales y profesionales, buscando elaborar y establecer en ellos relaciones entre las diferentes partes, como se autoproducen y cómo cambian, es decir, como se influyen como un todo. Por lo tanto, se requiere transformar la manera de pensar, en procura de la construcción de un pensamiento contextuado, globalizador, abierto y reflexivo.

Siendo las cosas así, resulta claro que la formación profesional del futuro medico requiere un cambio de paradigma que permita dar respuestas a las necesidades que se plantean en al ámbito de la salud. Por lo tanto, las universidades deben revisar sus objetivos y procurar replantear su misión orientada hacia la calidad educativa y la pertinencia social del conocimiento. Sin embargo, a pesar de que las universidades 
comprenden la necesidad de establecer los cambios pertinentes que conlleven a lograr transformaciones acordes con el momento histórico, permanecen estancadas en las mismas prácticas pedagógicas basadas en la transmisión de conocimientos fragmentados dando relevancia a lo memorístico.

Es por eso, que se deben propiciar cambios en los elementos fundamentales de la universidad, especialmente en lo que se refiere a su reestructuración académicoadministrativa, y en cuanto a lo que se corresponde al modelo académico, se debe incluir una revisión del mismo que involucre el análisis y evaluación de los componentes curriculares que propician la formación de un determinado tipo de profesional y orientar, además la vinculación de la universidad con su entorno(VIII Reunión Nacional de Currículo, 2008).

En este sentido, cabe poner de relieve que la educación basada en competencias está centrada en el estudiante, y su propósito se orienta al desarrollo del pensamiento crítico, con la intención de que éste cuente con herramientas que le permitan discernir de forma racional y oportuna una resolución pertinente a las diferentes situaciones problemáticas que le puedan surgir durante su ejercicio como profesional. De esta forma se tiene que con el enfoque de competencias, el estudiante produce un resultado y el docente se convierte en facilitador en la aprehensión de los saberes.

Es importante destacar, que este tipo de aprendizaje es primordial en la formación del estudiante de medicina, el cual debe demostrar conocimientos, habilidades, destrezas y competencias como futuro médico en el ámbito de la salud. Por otra parte, se debe señalar que existen diversas publicaciones que hacen referencia acerca de las competencias que debe tener el estudiante de medicina, específicamente en la práctica clínica. Estas publicaciones comenzaron a emerger en la literatura médica desde la década de los 70 y se orientaban a un cambio a nivel conductual y procedimental. En este sentido, Epstein (2007), definió:

La competencia en medicina como el uso habitual y juicioso de la comunicación, conocimiento, habilidades técnicas, razonamiento clínico, emociones y valores, que se reflejan en la práctica diaria para el beneficio de los individuos y las comunidades a las que 
sirven y que las competencias deben ser una práctica a lo largo de la vida.

El paradigma dominante en lo que se refiere a la educación médica se caracteriza por los siguientes aspectos: Centrado en el sistema educativo, currículo definido por los contenidos programáticos, evaluación mediante tareas, exámenes y evidencias fijadas por el plan de estudios .Pero debido a la creciente mundialización en la educación médica además de la interacción entre médicos de diferentes naciones, se plantea como necesario la revisión curricular en las universidades y la implementación de un currículo por competencias que permita al futuro médico contar con las herramientas y las capacidades necesarias para lograr el mayor desenvolvimiento en su área de trabajo, así como la efectiva y pertinencia de su actuación como profesional en cualquier circunstancia.

Es importante subrayar, que el ejercicio profesional del futuro médico requiere de una formación contextual, es por ello que la competencia a este nivel, permite reflejar la relación entre las habilidades del individuo y las actividades que desempeña en una situación particular dentro del mundo real(García, 2010). Entre los factores contextuales frecuentes que se destacan en la formación del estudiante de medicina están:

-El cuidado del paciente.

-El conocimiento médico.

-El aprendizaje y mejoría basada en la práctica.

-Las habilidades interpersonales y de comunicación.

-La ética y el profesionalismo.

-Las actividades prácticas basadas en el enfoque del sistema de salud.

Por su parte, se plantea que las competencias contextuales deben enriquecerse con la formación y desarrollo de otras, entre las que se incluyen: trabajo en equipo, liderazgo, uso racional de las tecnologías de la comunicación e información, ética en la educación médica, desarrollo profesional continuo propio, uso de una comunicación empática y asertiva(Zabalza, 2003). De esta manera, se busca orientar el aprendizaje hacia una 
formación integral y establecer como meta deseable entre todos los involucrados en el proceso de la educación del estudiante, el poder asegurar que los futuros médicos sean competentes en el área de la medicina en la que ejercerán la profesión.

Por consiguiente, los aportes que se proponen desde el enfoque de un diseño curricular por competencias es modificar la perspectiva que desde el punto de vista de la formación del futuro profesional en el área de la salud y específicamente el médico tienen las universidades, el cual está dirigido a la acumulación de conocimientos por un aprendizaje que se debe orientar al saber y al saber hacer contextualizado. En este sentido, las universidades desde el ámbito académico deben realizar una revisión del diseño curricular para adaptarlo e integrarlo a las nuevas expectativas que demandan la formación de profesionales con las capacidades, habilidades, destrezas y conocimientos en pertinencia con la realidad social. Es por ello, que Zabalza (2003), expone que;

\begin{abstract}
El diseño y desarrollo curricular basado en competencias constituye un modelo facilitador con múltiples beneficios, tanto para el sistema en su conjunto y en sus orientaciones, como también para algunos actores y elementos específicos del ámbito educacional. Un currículo orientado por el enfoque por competencias puede brindar la oportunidad histórica de abrir un gran espacio de reflexión y no de cerrarlo sobre los procesos de diseño curricular, las prácticas de enseñanza y las formas de evaluación que tienen lugar en la universidad.
\end{abstract}

De esta manera, se vislumbra que la universidad debe formar estudiantes no solo para la ejecución de ciertas acciones que corresponden con su ámbito laboral sino que debe formar profesionales integrales sustentados en la enseñanza para el saber y la enseñanza para el saber actuar y de esta forma desarrollar sus potencialidades, orientado todo esto hacia un profesional competente, capaz de actuar de forma eficaz de manera individual y colectiva como miembro importante de la sociedad y en la cual cumple un rol efectivo en la resolución de las problemáticas.

En esta perspectiva, se tiene que The American Society for Tropical Medicine and Hygiene Committee on Medical Education, en Estados Unidos (EUA,) han propuesto 
tres dominios de competencias que se recomiendan para todos los estudiantes de medicina tanto en EUA como en Canadá, los cuales incluyen:

-Dominio de competencia 1: Enfermedades globales; Se propone que todo estudiante debe tener un conocimiento básico de las principales enfermedades que afectan y matan a las personas a nivel mundial.

-Dominio de competencia 2: Medicina del viajero; Alrededor de 800 millones de personas en el mundo cruzaron fronteras internacionales en el 2005. Más de 28 millones de residentes de los EUA viajaron al extranjero en ese año y el $51 \%$ de ellos lo hizo a países en vías de desarrollo y más de un millón de esos viajeros regresaron con síntomas de alguna enfermedad.

-Dominio de competencia 3: salud del inmigrante; Se estima que al menos el $12 \%$ de los habitantes en EUA, nacieron fuera de ese país. El 77\% del millón de inmigrantes legales en los EUA proceden de países en vías de desarrollo. Además se debe agregar que por cada inmigrante legal, existen 11 ilegales y por otra parte la ciudad de Toronto lidera en América del Norte la estadística de población inmigrante, ya que casi el $43 \%$ de su población nació fuera de Canadá.

En consecuencia, con los resultados obtenidos, se implementaron en las universidades de Estados Unidos y Canadá donde se imparte la medicina como carrera y específicamente en el currículo, cursos de 30 horas en relación con la educación en salud global, de manera de actualizar y contextualizar los conocimientos y permitir el mejor desempeño del futuro médico.

Debe señalarse, que en relación a la profesión médica, una de las formulaciones recientes más aceptada universalmente establece como sus principios esenciales son:

- Primacía del bienestar del paciente.

- Respeto por la autonomía del mismo.

- Promoción de la justicia social.

Igualmente, la misma formulación describe las responsabilidades fundamentales de la profesión médica en la actualidad, relacionadas con:

- La competencia profesional. 
- La honestidad con el paciente.

- La confidencialidad.

- La adecuada relación médico-paciente.

- La calidad asistencial.

- La equidad en el acceso.

- La gestión racional y eficaz de los recursos.

- El conocimiento científico como base de la práctica.

- El mantenimiento de la confianza social a través de una adecuada gestión del conflicto de intereses.

- La autorregulación y el respeto mutuo entre colegas.

De tal manera, que se han establecido tres grandes ámbitos de competencias exigibles al médico de hoy: el conceptual o de pensamiento crítico (conocimientos),el técnico (habilidades) y el de la relación interpersonal (actitudes). Es por ello que estos tres ámbitos son los que universalmente se reconocen como componentes fundamentales de la competencia profesional del médico.(Colegio de Médicos Madrid, 2006)

En consecuencia, se tiene que la formación del médico del siglo XXI, implica mucho más allá que una exorbitante cantidad de conceptos y teorías, sino que debe involucrar una formación integral que le permita desenvolverse de forma efectiva y eficaz en el ámbito laboral y a través de su ejercicio profesional dar respuesta oportuna a la resolución de las problemáticas de salud de la población y poder implementar a través de estrategias, acciones para el control de las enfermedades emergentes y reemergentes además de las enfermedades desatendidas u olvidadas, las cuales se constituyen en un problema de salud pública latente.

Por lo tanto, se requiere de un profesional formado con calidad educativa y con pertinencia social del conocimiento para contribuir con la resolución de las problemáticas de salud, y poder así estar en consonancias con las metas fijadas por las organizaciones internacionales hacia una salud para todos a partir de la promoción de salud en los objetivos de desarrollo sostenibles de acuerdo a la agenda de la OMS 
. Revista Arbitrada Multidisciplinaria de Ciencias de la Salud. SALUD Y VIDA

Volumen 2. Número 4. Año 2. Julio - Diciembre 2018. Santa Ana de Coro, Venezuela

Hecho el depósito de Ley: FA2016000010

ISSN: $2610-8038$

FUNDACIÓN KOINONIA (F.K). Santa Ana de Coro, Venezuela.

Sandra Romero

2030. Además, el médico como profesional cruza frontera y su ejercicio debe estar en concordancia con las demandas y retos que plantea la sociedad del siglo XXI.

\section{REFERENCIAS CONSULTADAS}

1. Armus, D., (2000). La enfermedad en la historiografíade América Latina moderna.Swarthmore College, USA.

2. Becker, G. (2004). Capital humano y pobreza., En Consejo Pontificio para la Familia (Ed.),. Lexicón: términos ambiguos y discutidos sobre familia, vida y cuestiones éticas (pp. 87-92). Madrid: Palabra.

3. Benach, J., (2005). Aprender a mirar la salud. Barcelona: El Viejo Topo.

4. Borgia, F.,(2006). La Salud en América Latina. Rev. Medicina Social, volumen 1, número 3, diciembre 2006.

5. Borrell, C.,(2012).Epidemiologia social: la persona, la población y los determinantes sociales de la salud.Cuadernos de la fundación dr. Antonio Esteve no 32 .

6. Castro, R., (2015). Familia, Economía y Bien Común: efectos de la transición demográfica.Un acercamiento desde las ciencias sociales.[Consultado13octubre 2018]. Disponible en:https://www.researchgate.net/publication/286882922

7. Conferencia Regional de América Latina y el Caribe (2008).

8. Consejo de Colegios de Médicos (2006). Ser médico, hoy. Retos del nuevo profesionalismo médico en España. Madrid.

9. Coronel,J., (2017). La promoción de la salud: evolución y retos en América Latina.MEDISAN vol.21 no.7 Santiago de Cuba.

10. Dumont, G. (2004). Demografía, transición demográfica y política demográfica., En Consejo Pontificio para la Familia (Ed.),. Lexicón: términos ambiguos y discutidos sobre familia, vida y cuestiones éticas (pp. 193-201). Madrid: Palabra.

11. Espteins, M. (2007). Assement in Medical education. N Engl J Med.

12. García, J. (2010). Educación Médica Basada en Competencias. Medic Graphi. México. 
13. Krieger, N.,(2002). Glosario de epidemiologia social. RevPanam Salud Publica/Pan Am J Public Health.

14. Litman, T., (1994). The family as a basic unit in health. En: Donati P.Sociología de la Salud. Madrid. ed. Díaz de Santos. 1994 p. 186-90.

15. Longman, P., (2011). La cuna vacía. Cómo las tendencias familiares contemporáneas perjudicanla economía familiar. [Consultado 13 octubre 2018]. Disponible en:http://sustaindemographicdividend.org/wpcontent/uploads/2012/07/AF-Estudio-Social-Trends-Institute

16. Morandé, P., (2006). Familia y Sociedad contemporánea., En Consejo Episcopal Latinoamericano (Ed.), La Familia en América Latina: Desafíos y esperanzas. (pp. 8-22). Santiago Bogotá: Celam.

17. Morín, E. (2007). Los Siete Saberes Necesarios para la Educación del Futuro. UNESCO.

18. Organización Mundial de la Salud (2016). Novena Conferencia Mundial de Promoción de Salud. La promoción de la salud en los Objetivos de Desarrollo Sostenible. Shanghái: OMS; 2016 [Consultado 6 octubre 2018]. Disponible en: http://www.who.int/mediacentre/events/2016/health-promotion/es/

19. Organización Panamericana de la Salud (1992). Teoría y práctica de la salud pública. Documento Escuela de Salud Pública. La Habana.

20. Ramos, B., (2000). La nueva salud pública. Revista Cubana Salud Pública.

21. Ramos, B., (1990). Medicina social y salud pública en Cuba. La Habana. Editorial Pueblo y Educación.

22. UNESCO (1998). La Educación en el Siglo XXI. Visión y Acción, Informe de la Conferencia Mundial sobre Educación Superior.

23. VIII Reunión Nacional de Currículo y II Congreso Internacional de Calidad e Innovación en la Educación Superior (2008).

24. Whitehead, M.,(2006). Concepts and principlesfor tackling social inequities in health. Leveling up. World Health Organization. 
. Revista Arbitrada Multidisciplinaria de Ciencias de la Salud. SALUD Y VIDA

Volumen 2. Número 4. Año 2. Julio - Diciembre 2018. Santa Ana de Coro, Venezuela.

Hecho el depósito de Ley: FA2016000010

ISSN: $2610-8038$

FUNDACIÓN KOINONIA (F.K). Santa Ana de Coro, Venezuela.

Sandra Romero

25.Zabalza, M. (2003). La Enseñanza Universitaria. El escenario y sus Protagonistas. Editorial Narcea. Madrid. España.

C02018 por el autor. Este artículo es de acceso abierto y distribuido según los términos y condiciones de la licencia Creative Commons Atribución-NoComercial-Compartirlgual 4.0 Internacional (CC BY-NC-SA 4.0) (https://creativecommons.org/licenses/by-nc-sa/4.0/). 